\title{
NOTE ON DEFINING PROPERTIES OF HARMONIC FUNCTIONS
}

\author{
BY STANISLAS SAKS*
}

1. Introduction. The object of this note is to extend slightly, and to give new proofs for, two theorems characterizing harmonic functions. The first of these is the classical theorem discovered independently by Bôcher and Koebe. $\dagger$

Theorem A. If $u(x, y)$ is continuous with its partial derivatives of the first order in an open continuum $D$, and if for every circle $C$ contained in $D$

$$
\int_{C} \frac{\partial u}{\partial n} d s=0
$$

then $u(x, y)$ is harmonic in $D$.

The second is Gergen's recent generalization of the above theorem.t

TheOREM B. If $v(x, y)$ is harmonic and positive in $D$, if $u(x, y)$ is continuous with its partial derivatives of the first order in $D$, and if

$$
\int_{C}\left(v \frac{\partial u}{\partial n}-u \frac{\partial v}{\partial n}\right) d s=0
$$

for every circle $C$ contained in $D$, then $u(x, y)$ is harmonic in $D$.

We shall prove the following two theorems.

THEOREM 1. If $u(x, y)$ is continuous with its partial derivatives of the first order in $D$, and if for every point $(x, y)$ in $D$

$$
\lim _{r \rightarrow 0} \frac{1}{2 \pi r} \int_{0}^{2 \pi}\left[\frac{\partial u(x+r \cos \theta, y+r \sin \theta)}{\partial r}\right] d \theta=0,
$$

then $u(x, y)$ is harmonic in $D$.

* International Research Fellow. The author wishes to thank Professor O. D. Kellogg for criticisms.

$\dagger$ See, for instance, Kellogg, Foundations of Potential Theory, 1929, p. 227.

$\ddagger \mathrm{J}$. J. Gergen, Note on a theorem of Bôcher and Koebe, this Bulletin, vol. 37 (1931), pp. 591-596. 
THEOREM 2. If $v(x, y)$ is harmonic and different ${ }^{*}$ from 0 in $D$, if $u(x, y)$ is continuous with its partial derivatives of the first order in $D$, and if for every point $(x, y)$ in $D$

$$
\lim _{r \rightarrow 0} \frac{1}{2 \pi r} \int_{0}^{2 \pi}\left(v \frac{\partial u}{\partial r}-u \frac{\partial v}{\partial r}\right) d \theta=0
$$

(where $u=u(x+r \cos \theta, y+r \sin \theta)$, and similarly for $v)$, then $u(x, y)$ is harmonic in $D$.

2. Proof of Theorem 1. The last of these theorems evidently contains the first three as special cases. Its proof, however, depends on Theorem 1, and this is therefore established first. We shall use the following theorem of Blaschke, $\dagger$ generalizing the mean-value theorem for harmonic functions.

$A$ necessary and sufficient condition that the continuous function $u(x, y)$ be harmonic in $D$ is that at every point $(x, y)$ of $D$

$$
\lim _{r \rightarrow 0} \frac{1}{\pi r^{2}} \int_{0}^{2 \pi}[u(x+r \cos \theta, y+r \sin \theta)-u(x, y)] d \theta=0 .
$$

Theorem 1 follows at once. To see this, we write

$$
F(r)=\frac{1}{\pi r^{2}} \int_{0}^{2 \pi}[u(x+r \cos \theta, y+r \sin \theta)-u(x, y)] d \theta,
$$

and employ the well known law of the mean used for the evaluation of indeterminate forms. The result is

$$
F(r)=\frac{1}{2 \pi \rho} \int_{0}^{2 \pi}\left[\frac{\partial u(x+r \cos \theta, y+r \sin \theta)}{\partial r}\right]_{r=\rho} d \theta,
$$

where $0<\rho<r$. By the hypothesis of Theorem $1, \lim _{r \rightarrow 0} F(r)=0$. Hence, by Blaschke's theorem, $u(x, y)$ is harmonic $\ddagger$

* It would be sufficient to assume simply that $v$ is harmonic and not identically 0 in $D$.

$\dagger$ Ein Mittelwertsatz und eine kennzeichnende Eigenschaft des logarithmischen Potentials, Leipziger Berichte, vol. 63 (1916), pp. 3-7. See also the remarks of E. Hopf, Jahresbericht der Deutschen Mathematiker Vereiningung, vol. 39 (1930), Part 2, p. 5.

¥ The proof may also be given independently of Blaschke's theorem, by using the same method as is used there. 
3. Proof of Theorem 2. We turn to the proof of Theorem 2. The hypothesis permits us to write, for all sufficiently small $r$,

$$
\begin{aligned}
& v(x+r \cos \theta, y+r \sin \theta)= v(x, y)+\sum_{1}^{\infty} r^{n}\left(a_{n} \cos n \theta+b_{n} \sin n \theta\right), \\
& \begin{aligned}
& u(x+r \cos \theta, y+r \sin \theta)= u(x, y)+r\left(A_{1} \cos \theta+B_{1} \sin \theta\right) \\
&+r h(r, \theta), \\
&(3) \quad \\
& \frac{\partial v}{\partial r}=a_{1} \cos \theta+b_{1} \sin \theta+\sum_{2}^{\infty} n r^{n-1}\left(a_{n} \cos n \theta+b_{n} \sin n \theta\right), \\
& \frac{\partial u}{\partial r}=A_{1} \cos \theta+B_{1} \sin \theta+h^{\prime}(r, \theta),
\end{aligned}
\end{aligned}
$$

where $A_{1}, B_{1}, a_{n}, b_{n}$ are independent of $r$ and $\theta$, and where $h(r, \theta)$ and $h^{\prime}(r, \theta)$ tend to 0 with $r$, uniformly as to $\theta$.

If these expressions are substituted in (2), and the terms of degree higher than the first in $r$ omitted, together with those whose integrals vanish, the hypothesis of Theorem 2 becomes

$$
\lim _{r \rightarrow 0} \frac{1}{2 \pi r} v(x, y) \int_{0}^{2 \pi} \frac{\partial u(x+r \cos \theta, y+r \sin \theta)}{\partial r} d \theta=0 .
$$

As $v(x, y)$ is not 0 , this is equivalent to the hypothesis of Theorem 1. Hence, by the latter theorem, $u(x, y)$ is harmonic.

4. Conclusion. We remark that if in Theorems 1 and 2 we replace the sign of equality by $\geqq$ (for $v>0$, otherwise by $\leqq$ ), we obtain conditions which are necessary and sufficient that the function $u(x, y)$ be subharmonic in $D$. These results are obtained by an extension of Blaschke's theorem to the case of subharmonic functions. * The necessity of the condition may be proved easily by the method of integral means.

HARVARD UNIVERSITY

* S. Saks, On convex and subharmonic functions (in Polish), Mathesis Polska, vol. 6 (1931), pp. 43-66; and On subharmsnic functions, Acta Universitatis Hungaricae, vol. 5 (1932), pp. 187-193. 